\title{
PANORAMA DOS SISTEMAS E MODELOS CONTEMPORÂNEOS DE AVALIAÇÃO DE LIVROS ACADÊMICOS ${ }^{1}$
}

\begin{abstract}
Resumo: Situado no contexto da comunicação científica e da avaliação da produção acadêmica, o presente estudo propõe mapear e descrever os sistemas e modelos contemporâneos de avaliação de livros acadêmicos. Ao longo da pesquisa constatou-se que a avaliação da qualidade e do impacto dos livros encontra-se em situação ainda incipiente face à avaliação de artigos e periódicos científicos. No entanto, observaram-se alguns esforços no sentido de construir metodologias específicas para avaliar os livros produzidos no âmbito acadêmico. A fase inicial do estudo foi dedicada à identificação e ao mapeamento de plataformas, sistemas, aplicativos e outras iniciativas correntes, por meio de pesquisa documental de natureza exploratória durante a qual foram consultados especialistas, sistemas governamentais, ferramentas de busca, grupos de pesquisa, além da mineração e do exame de referências localizadas em documentos que remetiam a métodos de avaliação de livros. $\mathrm{O}$ exame desses documentos e de artigos publicados sobre o tema ensejou a elaboração de uma matriz de coleta de dados utilizada para organizar os sistemas e modelos identificados de acordo com um grupo de variáveis: tipologia da avaliação, técnicas utilizadas para reunir informações sobre as obras, produto gerado e tipo de impacto mensurado. Os resultados evidenciaram a existência de 24 modelos e sistemas de avaliação, a maioria envolvendo iniciativas comerciais, e outros resultantes de projetos de cientistas e grupos de pesquisa e de modelos institucionais e governamentais cujo principal interesse reside na avaliação da atividade científica de países e regiões. Embora apresentem variações nos indicadores aferidos e nos métodos de avaliação, a maioria (71\%) dos modelos se dedica essencialmente a avaliar indiretamente a qualidade e o impacto dos livros acadêmicos, tendência que dificulta a observação de outros aspectos característicos dos livros, que envolvem indicadores de natureza multidimensional, como os socioculturais.
\end{abstract}

Palavras-Chave: Livros acadêmicos. Avaliação da produção científica. Indicadores de575757 desempenho e impacto.
Universidade Federal do Espírito Santo (UFES). claudio.franca@ufes.br

Nanci Oddone

Doutora em Ciência da

Informação pela

Unversidade Federal do Rio

de Janeiro (UFRJ) neoddone@gmail.com

\section{OVERVIEW OF CONTEMPORARY SYSTEMS AND MODELS FOR THE EVALUATION OF ACADEMIC BOOKS}

\begin{abstract}
Situated in the context of scholarly communication and the evaluation of scientific production, this study proposes to map and describe contemporary systems and models for the evaluation of academic books. Throughout the research it was found that the evaluation of the quality and impact of books is still in an incipient situation compared to the evaluation of scientific articles and journals. However, some efforts were made to build specific methodologies to evaluate books produced in the academic field. The initial phase of the study was dedicated to the identification and
\end{abstract}

1 Este artigo é uma versão revista e ampliada de comunicação oral apresentada no GT7 do XIX ENANCIB, realizado em Florianópolis em 2019. 
mapping of platforms, systems, applications and other current initiatives, through documentary research of an exploratory nature during which specialists, government systems, search tools, research groups were consulted, in addition to mining and examining references located in documents that referred to book evaluation methods. The examination of these documents and of published articles on the subject gave rise to the elaboration of a data collection matrix used to organize the systems and models identified according to a group of variables: type of assessment, techniques used to gather information about the books, generated product and type of impact measured. The results showed the existence of 24 evaluation models and systems, most involving commercial initiatives, and others resulting from projects by scientists and research groups and from institutional and governmental models whose main interest lies in the evaluation of the scientific activity of countries and regions. Although there are variations in the indicators measured and in the evaluation methods, most $(71 \%)$ of the models are essentially dedicated to indirectly assessing the quality and impact of academic books, a trend that makes it difficult to observe other characteristic aspects of books, that involves indicators of multidimensional nature, such as socio-cultural.

Keywords: Scientific evaluation. Indicators of performance and impact. Scholarly books. 


\section{INTRODUÇÃO}

O desenvolvimento de metodologias e indicadores para mensurar e avaliar os produtos originários da atividade científica acompanha a história da ciência. Entre os séculos XVI e XVII, a literatura registra a adoção de práticas avaliativas prévias, em geral tomando como base a consulta a especialistas de notável conhecimento, que deliberavam sobre a credibilidade do método empregado e sobre a qualidade da produção. Evidencia-se, assim, a aproximação entre avaliação e observação de qualidade, binômio que desde então configurouse como princípio norteador da prática científica. Contudo, foi a partir do século XX que as práticas de mensuração e avaliação da ciência avançaram de forma significativa, influenciadas inicialmente por fatores geopolíticos, como as guerras mundiais e a polarização decorrente da Guerra Fria. Nesse período, registrou-se um crescimento exponencial da documentação científica, que demandou a adoção de critérios mais objetivos e céleres para avaliar o resultado das pesquisas, favorecendo a introdução dos métodos quantitativos de controle e análise da produção científica.

Adaptando-se ao modus operandi das ciências naturais no que tange aos padrões da comunicação científica, as atividades de avaliação baseadas em critérios quantitativos tomaram como fonte preferencial o periódico científico. A partir dos textos publicados nos periódicos produziram-se índices derivados da quantidade de citações recebidas por cada artigo. Mais adiante, esses índices se converteram em indicadores decisivos para fornecer subsídios à avaliação das atividades científicas. Com o avanço das tecnologias eletrônicas e o advento das bases de dados e das publicações em formato digital, novos métodos foram desenvolvidos para avaliar a produção científica, de modo que, atualmente, esses indicadores são empregados para avaliar a ciência como um todo, do gerenciamento de recursos financeiros até a produtividade da elite científica, incluindo o desempenho dos centros universitários e das instituições de pesquisa.

Não obstante muitos cenários possam ser vislumbrados a partir do modelo de análise que toma o periódico científico como fonte primeira para o fornecimento de dados de 
avaliação da atividade científica, outras fontes de informação, como os livros e as patentes, por exemplo, também são utilizadas para publicar descobertas científicas, convertendo-se, por vezes, nos principais canais de comunicação e divulgação de algumas áreas do conhecimento. Ao contrário do que ocorreu com o periódico, entretanto, a disseminação de conhecimentos e inovações científicas por meio dos livros subsistiu à margem dos sistemas de avaliação, talvez porque nas áreas em que a ciência normal prevalece, como observou Kuhn (2007), o livro caracteriza-se principalmente como "manual", isto é, como recurso didático que visa a reprodução do saber previamente validado pelos pares como fato científico comprovado (LATOUR, 1997).

A visão tradicional das práticas de avaliação da atividade científica alinha-se à perspectiva positivista de que apenas se pode avaliar a ciência e seus produtos pelo impacto científico, isto é, pela forma como uma produção científica reverbera entre os cientistas, sinalizado pela quantidade de citações recebidas. Todavia, no caso do livro, devido a seu caráter multidimensional, outros tipos de impacto poderiam ser aferidos, de acordo com a finalidade a que a obra se propõe. Desse modo, um livro pode impactar pelas dimensões didática, técnica, cultural e, inclusive, científica. Um caso exemplar de livro que alcançou ampla repercussão na comunidade científica da época em razão de seu espetacular poder de descoberta e inovação científica foi A origem das espécies, de Charles Darwin, publicado em 1859. ${ }^{2}$

O cenário de contínua expansão das iniciativas de avaliação da produtividade científica objetiva, entre outras ações, facilitar a tomada de decisão quanto às promoções na carreira, subsidiar a obtenção de financiamentos e sistematizar as avaliações institucionais, o que revela a necessidade de definir métodos e parâmetros específicos para avaliar os livros acadêmicos, atendendo às especificidades do tipo de informação que esses produtos editoriais veiculam e atribuindo-lhes métricas e indicadores que sinalizem sua qualidade e seu impacto. Os modelos e metodologias de avaliação da ciência devem garantir aos livros, portanto, escalas de valor que estejam em acordo com as diferentes dimensões de sua contribuição para

2 DARWIN, C. On the origin of species by means of natural selection. London: John Murray, 1859. 
a construção do conhecimento.

A conjuntura atual dos sistemas de avaliação de livros acadêmicos apresenta um panorama ainda incipiente, com predomínio de soluções comerciais, embora também se verifiquem esforços para desenvolver estratégias e padrões independentes, a partir de iniciativas e projetos coordenados por pesquisadores, por grupos de pesquisa e por órgãos governamentais interessados na avaliação das atividades de pesquisa de determinados países. Da mesma forma, na literatura, são reduzidas as publicações que se dedicam ao estudo do livro acadêmico e à sua avaliação.

Em vista do contexto delineado e considerando ainda a dispersão das práticas de apoio à avaliação dos livros acadêmicos, o presente estudo propõe-se a mapear os sistemas, plataformas, modelos, metodologias e instrumentos utilizados para avaliar a produção intelectual publicada por meio do livro acadêmico, classificando essas iniciativas de acordo com as tipologias de avaliação, as técnicas utilizadas para reunir informações sobre as obras, os produtos gerados e o tipo de impacto alcançado.

\section{NECESSIDADE DE DESENVOLVER PARÂMETROS PARA A AVALIAÇÃO DOS LIVROS ACADÊMICOS}

Desde meados do século XX, políticas e metodologias internacionais desenvolvidas para aferir a qualidade da produção científica popularizaram indicadores como o Fator de Impacto, as análises de citação e a revisão por pares que, sendo critérios relativamente objetivos, acabaram por ser estendidos e adotados para determinar também a qualidade dos periódicos acadêmicos enquanto veículos de circulação dessa produção. Embora os critérios criados por agências e empresas internacionais para avaliar os periódicos sejam claros e bem consolidados, adotá-los como referência para julgar a qualidade dos livros, ainda que apenas os acadêmicos, tem demonstrado ser uma tarefa no mínimo polêmica. No caso dos livros, a qualidade constitui um conceito complexo, sendo difícil traduzir esse conceito em indicadores quando muitas vezes o objetivo da verificação está estritamente associado à validação das 
políticas científicas de cada país (GIMÉNEZ-TOLEDO et al., 2016; GIMÉNEZ-TOLEDO; MAÑANA-RODRIGUEZ; TEJADA-ARTIGAS, 2015).

Acrescente-se a esse cenário o argumento de Luz (2005), no sentido de que os livros têm sido alvo de progressiva discriminação enquanto veículos de difusão de conhecimento. Para a autora, o sistema científico compõe-se de conjuntos hierárquicos regidos pela ótica da autoridade epistemológica das ciências naturais, que valoriza determinados critérios e padrões de publicação e disseminação, atribuindo reconhecimento e crédito aos autores que acatam tais ordenamentos. As consequências dessa conjuntura, institucionalizada pelas agências de avaliação e financiamento e também por universidades e centros de pesquisa, foi a desvalorização tácita do livro mesmo em áreas do conhecimento que tradicionalmente se valem dessas publicações como os principais meios de circulação e disseminação dos resultados das pesquisas (CAFÉ, 2012; CAFÉ; RIBEIRO; PONCZEK, 2018; RODRIGUES; ROSA; OLIVEIRA; BARROS, 2018; WHITLEY, 2011).

Como já assinalado, mesmo que as práticas de avaliação tenham como foco principal as pesquisas publicadas em periódicos, o livro ainda ocupa um locus expressivo no processo de comunicação científica, especialmente em áreas relacionadas às Humanidades e Ciências Sociais. Há diversas evidências na literatura demonstrando que os padrões de comunicação científica entre pesquisadores das Humanidades e das Ciências Sociais, de forma específica quanto à leitura e à citação, diferem do que se observa nas demais áreas do conhecimento (MEADOWS, 2009; MUELLER, 2005; TRZESNIAK, 2012). Assinalando o alto impacto que os livros chegam a ter na comunicação científica das áreas de Humanidades e Ciências Sociais, Hicks (2004) aponta a existência de quatro tipos de publicação usadas por esses cientistas: artigos em periódicos estrangeiros, livros, artigos em periódicos nacionais e textos em revistas não científicas. Além disso, essas comunidades apresentam características singulares, a exemplo da diversidade linguística, da predominância de temáticas locais e da importância atribuída às editoras nacionais, revelando-se numa dispersão maior de editoras quando se compara com a publicação em periódicos científicos (GIMÉNEZ-TOLEDO; MAÑANA-RODRIGUEZ; SIVERTSEN, 2017; GIMÉNEZ-TOLEDO; TEJADA-ARTIGAS; BORGES-DE-OLIVEIRA, 2019). Por esse motivo não se pode perder os livros de vista quando se trata de estabelecer critérios para qualificar a produção científica, pois isso 
resultaria na marginalização dos resultados de investigação publicados através desse importante veículo editorial (GIMÉNEZ-TOLEDO; MAÑANA-RODRIGUEZ; SIVERTSEN, 2017; GIMÉNEZ-TOLEDO; MAÑANA-RODRIGUEZ; TEJADA-ARTIGAS, 2015).

Na perspectiva dos pesquisadores em Humanidades e Ciências Sociais, portanto, os livros e capítulos de livros, mais do que os periódicos, constituem os veículos preferenciais para publicar sua produção (SANTOS; CABALLERO-RIVERO; SÁNCHEZ-TARRAGÓ, 2018). No entanto,

[...] independentemente de que no interior dessas comunidades a publicação de livros ou capítulos de livros tradicionalmente tenha trazido reconhecimento dos pares, a falta de indicadores de avaliação influi para que estes não sejam ponderados adequadamente. [...] Ainda quando os indicadores são considerados [...], quase sempre [...] sua pontuação é menor. O que se observa, então, é uma tendência a mudar práticas epistêmicas, privilegiando a produção de artigos, que é mais 'rentável' (SANTOS; CABALLERO-RIVERO; SÁNCHEZTARRAGÓ, 2018, p. 29)

Essas constatações confirmam a percepção de que, apesar da mudança nas formas de governança e de gerenciamento da atividade científica (WHITLEY, 2011), os livros permanecem sendo fortes vetores de produção e disseminação dos resultados de pesquisas, demonstrando a necessidade e até mesmo a urgência de desenvolver estratégias para avaliar o impacto e a qualidade dos livros. De fato, verifica-se atualmente uma compreensão mais consensual de que a singularidade desses tradicionais produtos editoriais deve ser considerada quando da definição de políticas e metodologias visando o estabelecimento de indicadores e métricas para avaliar a produção científica (THOMPSON, 2002).

As primeiras iniciativas voltadas à criação de procedimentos específicos para a avaliação de livros foram desenvolvidas por agências de fomento, em grande parte órgãos governamentais, cuja maior preocupação era avaliar a produção nacional e não propriamente observar o impacto das obras, fosse esse impacto científico, social ou cultural (TORRESSALINAS; ROBINSON-GARCIA; GORRAIZ, 2017). A esse grupo de iniciativas vieram agregar-se propostas de avaliação delineadas por grupos de pesquisadores interessados em 
compreender a dinâmica social do livro, bem como seu papel no processo de comunicação científica.

$\mathrm{Na}$ perspectiva dos estudos métricos, muito embora em 1934 Paulo Otlet tenha definido a bibliometria como uma atividade que se ocupava da mensuração ou quantificação dos livros (MOMESSO; NORONHA, 2017), até recentemente observou-se uma singular despreocupação com sua avaliação objetiva. Nesse sentido, ao comentar sobre a relevância e o alcance do livro como canal de disseminação da informação ao longo da história, Zuccala e Robinson-Garcia (2019) assinalam que até a década de 1970 havia uma cultura voltada ao monitoramento da qualidade dos livros por meio de resenhas publicadas em jornais impressos e, eventualmente, em periódicos científicos. Outro fator que contribuiu para o desinteresse da comunidade científica em projetar métricas que garantissem crédito ao valor e à influência dos livros publicados foi a ausência dos mesmos nas bases de dados comerciais que forneciam indicadores de avaliação de periódicos, já que a qualidade do livro não se encontrava associada à atribuição de indicadores quantitativos.

A partir da década de 1990, entretanto, surgiram esforços para que as técnicas quantitativas originárias dos estudos bibliométricos de periódicos pudessem aplicar-se ao universo dos livros acadêmicos. Eugene Garfield (1996), por exemplo, sugeriu a criação de um índice de citação específico para livros, argumentando que a ausência de uma ferramenta dessa natureza levava os pesquisadores a buscarem os índices de periódicos para verificar possíveis citações a seus livros:

Na perspectiva do cientista social ou do intelectual de Humanidades, a não inclusão de livros como fontes [citantes] nos índices de citação do ISI [Instituto de Informação Científica] pode ser um inconveniente quando se trata de extrair conclusões sobre o impacto de certos trabalhos. No entanto, a inclusão de livros como referências citadas nos índices de citação do ISI permitiu que o estudo dos livros mais citados fosse aceito como substituto razoável para estudos mais abrangentes, que poderiam incluir os livros como fontes [citantes]. A criação de um Índice de Citação de Livros constitui, sem dúvida, um grande desafio para o futuro e seria um subproduto a se esperar das novas mídias eletrônicas com capacidade de oferecer hiperligações! [...] (GARFIELD, 1996, p. 21). 
Apesar de idealizado em 1996, o índice de citação de livros descrito por Garfield só se tornou realidade nos últimos dez anos, com o surgimento de dois instrumentos formais, construídos especificamente para fornecer indicadores e métricas para livros com base no número de referências citadas: o Book Citation Index (BKCI), lançado no ano de 2011 pela editora Thomson Reuters como parte integrante da Web of Science e, no ano de 2013, o Books Expansion Project, integrado à base bibliográfica Scopus, da editora Elsevier. Tais ferramentas nasceram em meio à ampliação das discussões em torno da necessidade de acompanhar o impacto dos livros como instrumentos de difusão do conhecimento, não só acadêmico, mas cultural e social.

Em decorrência dessas primeiras iniciativas comerciais e das já mencionadas propostas institucionais ou de grupos de pesquisa e também das novas perspectivas de monitoramento viabilizadas pela web, o quadro de modelos e sistemas voltados à avaliação de livros, embora ainda incipiente, alargou-se bastante, demandando a realização de um mapeamento cuidadoso para identificar as características, as práticas e as metodologias adotadas nesses sistemas ou ferramentas, elementos que constituem o objeto desta pesquisa.

\section{ESTRATÉGIA METODOLÓGICA}

O estudo proposto insere-se na categoria de pesquisa descritiva e, para sua consecução, empregou-se abordagem qualitativa, motivada pela necessidade de conhecer a estrutura singular desses modelos e sistemas, identificando suas facetas, similaridades e heterogeneidades.

O primeiro estágio do estudo teve como universo de pesquisa os documentos que descrevem os sistemas de avaliação de livros acadêmicos. As fontes que subsidiaram esse estágio foram, predominantemente, os artigos de periódicos, embora tenham sido usados também documentos de natureza administrativa, como normas, diretrizes e roteiros empregados para subsidiar os processos de avaliação científica de instituições e órgãos governamentais. A fase inicial de exploração da literatura ocorreu em bases de dados 
referenciais e ferramentas de buscas nacionais e estrangeiras, tendo por objetivo recuperar evidências que remetessem aos métodos de avaliação de livros. As consultas tiveram início em março de 2018 e se estenderam até agosto do mesmo ano. Na oportunidade, delineou-se uma estratégia que contemplasse os termos de maior relevância para o estudo, ou seja, indicador, avaliação e livros acadêmicos. No caso da literatura estrangeira adotou-se o idioma inglês como padrão, utilizando os termos indicator, assessment, evaluation, scholarly book e academic book.

Para o levantamento da literatura nacional a busca foi realizada no Catálogo de Teses e Dissertações da Capes, na Base de Dados em Ciência da Informação (BRAPCI) e no Google Acadêmico, enquanto para recuperar artigos na literatura estrangeira optou-se pelas bases de dados referenciais Scopus; Library, Information Science and Technology Abstracts (LISTA); Library and Information Science Abstracts (LISA) e Google Scholar. Visando permitir a análise prévia dos registros recuperados para eliminar duplicações e selecionar os artigos potencialmente relevantes foram examinados o título, as palavras-chave e o resumo dos artigos.

Como etapa complementar ao levantamento primário procedeu-se à análise das referências citadas nos documentos recuperados, à qual se seguiu o exame das referências encontradas nas bibliografias disponíveis nos sítios das instituições e grupos de pesquisa identificados. Esse estágio foi fecundo, pois possibilitou o contato com um número considerável de propostas de avaliação de livros que, embora constituíssem apenas iniciativas dispersas e particulares de grupos ou de pesquisadores, sinalizavam concepções emergentes e modelos inovadores para avaliar a produção científica comunicada através dos livros.

A caracterização, propriamente dita, de cada uma dessas iniciativas se deu por meio da técnica de análise documental, que permitiu identificar as particularidades de cada proposta, evidenciando possíveis relações e associações entre seus princípios e metodologias. Os dados coletados fizeram parte de um estudo mais amplo que teve como objetivo mapear as iniciativas contemporâneas de avaliação de livros acadêmicos ${ }^{3}$ e que utilizou como

3 FRANÇA, C. M. Avaliação de livros acadêmicos: mapeamento dos modelos e indicadores contemporâneos. Dissertação defendida no Programa de Pós-graduação em Biblioteconomia da Universidade Federal do Estado do Rio de Janeiro em 2019. 
instrumento de coleta de dados uma matriz contendo onze variáveis (FRANÇA, 2019). Para o presente artigo foram selecionadas quatro categorias analíticas integrantes da referida matriz: tipologia da avaliação, técnicas utilizadas para reunir informações sobre as obras, produtos gerados e tipo de impacto mensurado, pois acredita-se que esses dados permitem delinear de maneira abrangente o panorama das iniciativas de avaliação de livros disponíveis atualmente.

\section{RESULTADOS}

A busca em bases de dados referenciais e em sítios de instituições e grupos de pesquisa possibilitou localizar um total de 43 iniciativas de avaliação de livros. Após o exame das fontes documentais foi possível selecionar 24 diferentes modelos de avaliação de livros acadêmicos, descritos de forma sumária no Quadro 1. Essas iniciativas incluem plataformas comerciais, sistemas institucionais constituídos para alocação de recursos, bem como para a avaliação da produção científica em um país ou região e, por fim, modelos conceituais, desenvolvidos por grupos de pesquisa ou pesquisadores individuais, com a finalidade de lançar um olhar crítico sobre o conhecimento registrado e disseminado nos livros acadêmicos.

Quadro 1: Descrição dos modelos/sistemas de avaliação de livros acadêmicos

\begin{tabular}{|l|l|}
\hline $\begin{array}{l}\text { Sistema ou } \\
\text { Modelo de } \\
\text { Avaliação }\end{array}$ & Descrição \\
\hline Altmetric & $\begin{array}{l}\text { Ferramenta que captura as interações individuais, monitorando e coletando a atenção } \\
\text { em mídias sociais para construir uma imagem mais completa sobre o alcance, } \\
\text { engajamento e influência das publicações. }\end{array}$ \\
\hline $\begin{array}{l}\text { Amazon } \\
\text { Reviews }\end{array}$ & $\begin{array}{l}\text { Sistema de avaliação de compras que, por meio das experiências anteriores obtidas } \\
\text { por outros usuários compradores, possibilita avaliar a qualidade da obra disponível } \\
\text { para venda. }\end{array}$ \\
\hline
\end{tabular}




\begin{tabular}{|c|c|}
\hline BFI & $\begin{array}{l}\text { Modelo desenvolvido na Dinamarca a fim auxiliar na distribuição de verbas para } \\
\text { alocação em pesquisas no âmbito das universidades dinamarquesas. }\end{array}$ \\
\hline Bipublishers & $\begin{array}{l}\text { Sistema desenvolvido para fornecer novos indicadores de base bibliométrica e } \\
\text { análise de desempenho dos editores acadêmicos, contemplando pesquisas } \\
\text { disseminadas em livros e capítulos de livros. }\end{array}$ \\
\hline $\begin{array}{l}\text { Book } \\
\text { Citation } \\
\text { Index }\end{array}$ & $\begin{array}{l}\text { Ferramenta com objetivo de monitorar o impacto científico por meio da contagem de } \\
\text { citações recebida por livros e capítulos de livros nos índices na base de dados } \\
\text { referencial Web of Science. }\end{array}$ \\
\hline $\begin{array}{l}\text { Book } \\
\text { Publisher } \\
\text { Library } \\
\text { Metrics }\end{array}$ & $\begin{array}{l}\text { Índice bibliométrico que busca medir a difusão e a visibilidade de editoras de livros } \\
\text { acadêmicos no âmbito das ciências humanas e sociais a partir da contagem dos livros } \\
\text { inclú́dos em catálogos de bibliotecas universitárias espanholas que integram o } \\
\text { catálogo coletivo da Red de Bibliotecas Universitárias (REBIUN). }\end{array}$ \\
\hline Bookmetrix & $\begin{array}{l}\text { Ferramenta web implementada pela editora Springer em colaboração com a empresa } \\
\text { Altmetric.com, cujo objetivo é monitorar as métricas de impacto de um determinado } \\
\text { livro ou capítulo de livro. }\end{array}$ \\
\hline CEA & $\begin{array}{l}\text { Sistema de certificação para promover a qualidade das edições de caráter científico } \\
\text { disseminadas em livros e, ao mesmo tempo, demonstrar as boas práticas adotadas } \\
\text { pelas editoras acadêmicas. }\end{array}$ \\
\hline Choice & $\begin{array}{l}\text { Banco de dados que disponibiliza resenhas de livros acadêmicos elaboradas por } \\
\text { especialistas e bibliotecários em diferentes áreas. }\end{array}$ \\
\hline Cristin & $\begin{array}{l}\text { Sistema desenvolvido na Noruega para conter os dados de pesquisa de instituições } \\
\text { públicas daquele país. }\end{array}$ \\
\hline FFLS & $\begin{array}{l}\text { Sistema de certificação desenvolvido na Finlândia que atribui selo de qualidade } \\
\text { indicando editoras acadêmicas de livros e periódicos científicos que adotaram } \\
\text { padrões de qualidade no processo de revisão por pares. }\end{array}$ \\
\hline $\begin{array}{l}\text { Google } \\
\text { Books }\end{array}$ & $\begin{array}{l}\text { Biblioteca eletrônica com acesso à literatura disseminada em livros, configurando-se } \\
\text { como um repositório para acessar pesquisas de forma integral ou parcial. }\end{array}$ \\
\hline $\begin{array}{l}\text { Google } \\
\text { Scholar }\end{array}$ & $\begin{array}{l}\text { Ferramenta de busca à literatura científica disseminada em vários canais de } \\
\text { informações, dentre eles livros e capítulos de livros que indica a quantidade de } \\
\text { citações recebidas. }\end{array}$ \\
\hline Goodreads & $\begin{array}{l}\text { Ferramenta que permite aos usuários pesquisar livremente dados sobre livros, } \\
\text { anotações, resenhas. }\end{array}$ \\
\hline GRPC & $\begin{array}{l}\text { Sistema de atribuição de selo de qualidade no qual se certifica que um procedimento } \\
\text { de revisão por pares avaliou positivamente uma publicação. }\end{array}$ \\
\hline JUFO & $\begin{array}{l}\text { Sistema desenvolvido para apoiar a avaliação da qualidade do resultado de pesquisas } \\
\text { em universidades e institutos de pesquisa da Finlândia. }\end{array}$ \\
\hline Kriterium & $\begin{array}{l}\text { Sistema de certificação que confere selos de qualidade para livros acadêmicos } \\
\text { suecos. Para obtenção do selo, as publicações passam por uma rigorosa revisão de } \\
\text { pares. }\end{array}$ \\
\hline $\begin{array}{l}\text { Library } \\
\text { Catalog } \\
\text { Analysis }\end{array}$ & $\begin{array}{l}\text { Modelo conceitual de medição do impacto da publicação a partir da aplicação de } \\
\text { técnicas bibliométricas a um conjunto de catálogos online de bibliotecas } \\
\text { universitárias. }\end{array}$ \\
\hline Libcitations & $\begin{array}{l}\text { Modelo conceitual de medição do impacto da publicação de acordo com o número } \\
\text { de bibliotecas que possuem determinado livro registrado em um catálogo. }\end{array}$ \\
\hline Plum & Ferramenta que fornece dados sobre o uso e impacto de pesquisa de produtos \\
\hline
\end{tabular}




\begin{tabular}{|l|l|} 
Analytics & científicos, conjugando métricas de citação e métricas recuperadas no ambiente web. \\
\hline $\begin{array}{l}\text { Publisher } \\
\text { Scholar } \\
\text { Metrics }\end{array}$ & $\begin{array}{l}\text { Índice bibliométrico desenvolvido para medir o impacto das editoras de livros } \\
\text { acadêmicos a partir da contagem de citaçães dos livros de autoria de docentes e } \\
\text { pesquisadores de universidades públicas espanholas. }\end{array}$ \\
\hline $\begin{array}{l}\text { Qualis } \\
\text { Livros }\end{array}$ & $\begin{array}{l}\text { instrumento de avaliação desenvolvido para mensurar a qualidade da obra } \\
\text { considerando critérios como circulação, gestão editorial e impacto na área. }\end{array}$ \\
\hline Scopus & $\begin{array}{l}\text { Base referencial que provê acesso a dados bibliográficos de uma série de fontes de } \\
\text { informação, dentre elas livros e capítulos de livros. }\end{array}$ \\
\hline $\begin{array}{l}\text { Scholarly } \\
\text { Publishers } \\
\text { Indicators }\end{array}$ & $\begin{array}{l}\text { Sistema de avaliação desenvolvido para estabelecer indicadores de processos de } \\
\text { avaliação e permitir a determinação de critérios sobre o conceito de prestígio } \\
\text { editorial. }\end{array}$ \\
\hline
\end{tabular}

Fonte: dados da pesquisa (2019).

As tipologias de avaliação adotadas pelos modelos e sistemas selecionados apresentaram variações quanto ao modo como os dados são gerados, coletados e interpretados. Conforme demonstrado no Quadro 2, observou-se a presença de três tipos de metodologia de avaliação: quantitativas, qualitativas e mistas.

Quadro 2: Tipologia da avaliação de livros acadêmicos

\begin{tabular}{|l|l|l|l|}
\hline Sistema ou Modelo de Avaliação & \multicolumn{3}{|c|}{ Tipologia de avaliação } \\
& Quantitativa & \multicolumn{1}{|c|}{ Qualitativa } & \multicolumn{1}{|c|}{ Mista } \\
\hline Altmetric & & & $\sqrt{ }$ \\
\hline Amazon Reviews & & $\sqrt{ }$ & \\
\hline BFI & $\sqrt{ }$ & & \\
\hline Bipublishers & $\sqrt{ }$ & & $\sqrt{ }$ \\
\hline Book Citation Index & $\sqrt{ }$ & & \\
\hline Book Publisher Library Metrics & & $\sqrt{ }$ & \\
\hline Bookmetrix & & $\sqrt{ }$ & \\
\hline CEA & & $\sqrt{ }$ & \\
\hline Choice & & $\sqrt{ }$ \\
\hline Cristin & & & \\
\hline FFLS & & & \\
\hline
\end{tabular}




\begin{tabular}{|l|l|l|l|}
\hline Google Books & $\sqrt{ }$ & & \\
\hline Google Scholar & $\sqrt{ }$ & & $\sqrt{ }$ \\
\hline Goodreads & & $\sqrt{ }$ & \\
\hline GRPC & & $\sqrt{ }$ & \\
\hline JUFO & & $\sqrt{ }$ & \\
\hline Kriterium & $\sqrt{ }$ & & \\
\hline Library Catalog Analysis & $\sqrt{ }$ & & \\
\hline Libcitations & & $\sqrt{ }$ & \\
\hline Plum Analytics & $\sqrt{ }$ & $\sqrt{ }$ & \\
\hline Publisher Scholar Metrics & & $\sqrt{ }$ & \\
\hline Qualis Livros & $\sqrt{ }$ & & \\
\hline Scopus & & & \\
\hline Scholarly Publishers Indicators & & & \\
\hline
\end{tabular}

Fonte: dados da pesquisa (2019).

Considerou-se como critério para classificação na tipologia quantitativa os modelos que propõem avaliar a qualidade dos livros acadêmicos tendo como parâmetro a quantidade de citações recebidas por essas obras, a ocorrência de exemplares em catálogos de bibliotecas e a atribuição de valorações disponíveis em uma escala de representação gráfica, simbolizada por estrelas.

O fundamento para a identificação da tipologia de caráter qualitativo envolveu a apreciação prévia como elemento norteador para avaliar a qualidade dos livros acadêmicos. A opinião de especialistas, a observação crítica de características editoriais das obras ou do prestígio do editor e a redação de resenhas foram as características preponderantes desses modelos.

Quanto à tipologia mista de avaliação, considerou-se serem aquelas que se dedicam a avaliar a qualidade dos livros acadêmicos por meio da conjugação entre o tratamento quantitativo e o qualitativo, reunindo o monitoramento das citações recebidas à existência e à relevância de resenhas, bookmarks e postagens em blogs. Nesse universo das metodologias 
mistas, destaca-se a popularização das mídias sociais, que suscitou o surgimento das métricas alternativas, ferramentas que auxiliam na avaliação do impacto social da produção científica, despontando como uma possibilidade de ampliar seu horizonte.

A observação dos modelos e sistemas de avaliação visando identificar as técnicas utilizadas para coletar informações sobre os livros publicados possibilitou distinguir duas diferentes abordagens: a técnica direta, que extrai informações com consulta direta às obras publicadas, analisadas de forma integral ou parcial; e a técnica indireta, que emprega elementos intermediários ou instrumentos complementares para deduzir a qualidade dos livros acadêmicos. Neste último caso a avaliação do conteúdo se efetiva pelo contato indireto com as obras publicadas, envolvendo dados de citação disponíveis em índices bibliográficos, incidência em catálogos de bibliotecas, prestígio da editora acadêmica que publicou a obra, nível de audiência e engajamento em redes sociais. O Quadro 3 relaciona os modelos e sistemas de avaliação às técnicas adotadas para reunião das informações.

Quadro 3: Técnicas utilizadas para reunir informações sobre as obras

\begin{tabular}{|c|c|c|}
\hline \multirow[t]{2}{*}{ Modelo de avaliação } & \multicolumn{2}{|c|}{ Tipo de técnica } \\
\hline & Direta & Indireta \\
\hline Altmetric & & $\sqrt{ }$ \\
\hline Amazon Reviews & $\sqrt{ }$ & \\
\hline BFI & & $\sqrt{ }$ \\
\hline Bipublishers & & $\sqrt{ }$ \\
\hline Book Citation Index & & $\sqrt{ }$ \\
\hline Book Publisher Library Metrics & & $\sqrt{ }$ \\
\hline Bookmetrix & & $\sqrt{ }$ \\
\hline CEA & & $\sqrt{ }$ \\
\hline Choice & $\sqrt{ }$ & \\
\hline Cristin & & $\sqrt{ }$ \\
\hline
\end{tabular}




\begin{tabular}{|c|c|c|}
\hline FFLS & $\sqrt{ }$ & \\
\hline Google Books & & $\sqrt{ }$ \\
\hline Google Scholar & & $\sqrt{ }$ \\
\hline Goodreads & $\sqrt{ }$ & \\
\hline GRPC & $\sqrt{ }$ & \\
\hline JUFO & & $\sqrt{ }$ \\
\hline Kriterium & $\sqrt{ }$ & \\
\hline Library Catalog Analysis & & $\sqrt{ }$ \\
\hline Libcitations & & $\sqrt{ }$ \\
\hline Plum Analytics & & $\sqrt{ }$ \\
\hline Publisher Scholar Metrics & & $\sqrt{ }$ \\
\hline Qualis Livros & $\sqrt{ }$ & \\
\hline Scopus & & $\sqrt{ }$ \\
\hline Scholarly Publishers Indicators & & $\sqrt{ }$ \\
\hline
\end{tabular}

Fonte: dados da pesquisa (2019).

Por um lado, o cenário delineado pela reunião desses dados evidencia a prevalência no uso de técnicas indiretas, sugerindo que seu emprego tende a tornar os processos de avaliação mais objetivos e padronizados e, por consequência, menos sujeitos aos vieses próprios das formas de avaliação realizadas por agentes humanos, que sofrem a influência de aspectos subjetivos. Por outro lado, acredita-se que os processos de produção e comunicação da ciência não podem basear-se exclusivamente em informações indiretas, sob pena de distanciar-se do secular padrão da avaliação científica, materializado pela revisão por pares, que mostra o valor atribuído pela ciência ao julgamento de outros pesquisadores para reconhecer as várias facetas da qualidade e do impacto da produção científica.

No que se refere aos produtos resultantes do processo avaliativo, verificou-se que as iniciativas estudadas representam o resultado da avaliação dos livros de diversas maneiras. 
Assim, embora se tenha observado a prevalência de produtos baseados em cálculos quantitativos, expressos através de conceitos numéricos, registrou-se igualmente a adoção de outros tipos de representação, como as notações alfanuméricas, logomarcas e recomendações, como se pode ver no Quadro 4.

Quadro 4: Produtos gerados pelos modelos/sistemas de avaliação de livros

\begin{tabular}{|l|l|}
\hline Modelo de avaliação & Produto Gerado \\
Altmetric & $\begin{array}{l}\text { Altmetric Donut utilizado para demonstrar o nível de atividade online em } \\
\text { torno de um resultado de pesquisa. }\end{array}$ \\
\hline Amazon Reviews & $\begin{array}{l}\text { Estrelas que interpretam uma avaliação quantitativa e resenhas } \\
\text { descrevendo as especificidades do documento, seguindo um viés } \\
\text { qualitativo. }\end{array}$ \\
BFI & $\begin{array}{l}\text { Indicador numérico contendo os pontos obtidos por uma editora a partir } \\
\text { da avaliação de especialistas. }\end{array}$ \\
Book Citation Index & $\begin{array}{l}\text { Métricas que refletem o total de livros publicados, total de capítulos de } \\
\text { livros publicados, total de citações recebidas, citações normalizadas, } \\
\text { índice de atividade da editora e porcentagem dos itens editados. }\end{array}$ \\
\hline Book Publisher Library & $\begin{array}{l}\text { Métricas relacionadas à atividade da editora: número de documentos } \\
\text { integrantes de um catálogo de biblioteca, total de inclusões de obras de } \\
\text { uma editora em uma biblioteca e média de inclusões. }\end{array}$ \\
\hline Metrics & $\begin{array}{l}\text { Métricas que reúnem o total de citações, além das atividades medidas em } \\
\text { mídias sociais e bases de dados, como o número de downloads, } \\
\text { compartilhamentos, curtidas e registros em bookmarks. }\end{array}$ \\
\hline Bookmetrix & $\begin{array}{l}\text { Relo de qualidade veiculado em livros de uma editora acadêmica. } \\
\text { o público-alvo a que se destina. }\end{array}$ \\
\hline CEA & $\begin{array}{l}\text { Indicador numérico representando a categoria de qualidade que a editora } \\
\text { acadêmica alcançou diante do painel de avaliadores. }\end{array}$ \\
\hline Choice &
\end{tabular}




\begin{tabular}{|c|c|}
\hline FFLS & Selo de qualidade em nível do editor, veiculado na folha de rosto da obra. \\
\hline Google Books & $\begin{array}{l}\text { Não apresenta uma métrica. Pelo emprego de ferramentas de } \\
\text { monitoramento webométrico é possível mensurar o número de citações } \\
\text { recebidas. }\end{array}$ \\
\hline Google Scholar & Índice contendo o número de citações. \\
\hline Goodreads & $\begin{array}{l}\text { Estrelas que interpretam uma avaliação quantitativa e resenhas } \\
\text { descrevendo as especificidades do documento, seguindo um viés } \\
\text { qualitativo. }\end{array}$ \\
\hline GRPC & Selo de qualidade em nível de obra, veiculado no colofão do livro. \\
\hline JUFO & $\begin{array}{l}\text { Indicador numérico representando a categoria de qualidade que a editora } \\
\text { acadêmica alcançou diante do painel de avaliadores. }\end{array}$ \\
\hline Kriterium & Selo de qualidade em nível de obra, veiculado na capa do livro. \\
\hline $\begin{array}{l}\text { Library Catalog } \\
\text { Analysis }\end{array}$ & $\begin{array}{l}\text { Conjunto de métricas para demonstrar a presença de editoras acadêmicas } \\
\text { da área de economia em catálogos de bibliotecas. }\end{array}$ \\
\hline Libcitations & Total de bibliotecas que possuem um exemplar de uma obra. \\
\hline Plum Analytics & $\begin{array}{l}\text { Infográfico Plum Print que demonstra as categorias das métricas } \\
\text { coletadas: uso, capturas, menções, mídias sociais e citações. }\end{array}$ \\
\hline $\begin{array}{l}\text { Publisher Scholar } \\
\text { Metrics }\end{array}$ & $\begin{array}{l}\text { Escala que varia entre } 0 \text { e } 100 \text {, construída pela multiplicação entre o total } \\
\text { de citações recebidas e número de livros de uma editora que figuram entre } \\
\text { os mais citados. }\end{array}$ \\
\hline Qualis Livros ${ }^{4}$ & $\begin{array}{l}\text { Estratos que representam a totalidade de pontos recebidos na avaliação. } \\
\text { Os indicadores variam de L1 a L4, sendo este último o que indica maior } \\
\text { pontuação. }\end{array}$ \\
\hline Scopus & Índice contendo o número de citações. \\
\hline $\begin{array}{l}\text { Scholarly Publishers } \\
\text { Indicators }\end{array}$ & $\begin{array}{l}\text { Indicadores que avaliam três dimensões das editoras acadêmicas: } \\
\text { prestígio, especialidades e requisitos adotados para a seleção de originais. }\end{array}$ \\
\hline
\end{tabular}

Fonte: dados da pesquisa (2019).

4 O roteiro original, construído pela Capes e tornado público em 2009 visando a avaliação trienal 2007-2009, estabeleceu quatro estratos de avaliação. Contudo uma nova proposta para avaliação de livros foi elaborada para aplicação no quadriênio 2017-2020 e nela foi acrescido mais um estrato, variando, doravante, entre L1 e L5, além das obras não classificadas, identificadas como LNC (BRASIL, 2019). 
Após a identificação do produto gerado, prospectou-se sobre o tipo de impacto que estava sendo mensurado em cada iniciativa de avaliação. Para essa análise adotou-se o enquadramento proposto por Wilsdon e outros (2015), que tipificaram o impacto produzido pelas pesquisas acadêmicas em duas categorias: o impacto acadêmico-científico e o impacto social.

No âmbito do impacto acadêmico-científico consideraram-se todas as iniciativas que avaliam o reconhecimento e a repercussão dos livros dentro dos limites do campo acadêmico, explorando métricas e indicadores que influenciam a avaliação de um pesquisador ou de uma instituição, a decisão editorial sobre a publicação de uma obra ou o mérito de um projeto visando seu financiamento. O impacto social, ao contrário, compreende o reconhecimento e a repercussão dos livros que ultrapassam o cenário acadêmico, incidindo em práticas relacionadas à educação, ao desenvolvimento social, à cultura e à economia. Exemplos desse tipo de impacto envolvem o uso de resenhas no desenvolvimento de coleções de bibliotecas, a experiência da qualidade editorial das obras tal como registrada por público não especializado em ferramentas de recomendação de leituras e em sistemas de avaliação de compras e a recomendação de obras para adoção como bibliografia básica de disciplinas universitárias, manifestando sua qualidade didática. O Quadro 5 apresenta os tipos de impacto identificados nos modelos e sistemas de avaliação de livros estudados nesta pesquisa.

Quadro 5: Tipo de impacto mensurado pelos modelos/sistemas de avaliação de livros

\begin{tabular}{|l|l|l|}
\hline Modelo de avaliação & \multicolumn{2}{|c|}{ Tipo de impacto mensurado } \\
& Impacto científico & \multicolumn{1}{|c|}{ Impacto social } \\
\hline Altmetric & & $\sqrt{ }$ \\
Amazon Reviews & & $\sqrt{ }$ \\
\hline BFI & $\sqrt{ }$ & \\
Bipublishers & $\sqrt{ }$ & \\
\hline Book Citation Index & $\sqrt{ }$ & \\
\hline
\end{tabular}




\begin{tabular}{|c|c|c|}
\hline Book Publisher Library Metrics & $\sqrt{ }$ & \\
\hline Bookmetrix & & $\sqrt{ }$ \\
\hline CEA & $\sqrt{ }$ & \\
\hline Choice & & $\sqrt{ }$ \\
\hline Cristin & $\sqrt{ }$ & \\
\hline FFLS & $\sqrt{ }$ & \\
\hline Google Books & $\sqrt{ }$ & \\
\hline Google Scholar & $\sqrt{ }$ & \\
\hline Goodreads & & $\sqrt{ }$ \\
\hline GRPC & $\sqrt{ }$ & \\
\hline JUFO & $\sqrt{ }$ & \\
\hline Kriterium & $\sqrt{ }$ & \\
\hline Library Catalog Analysis & $\sqrt{ }$ & \\
\hline Libcitations & & $\sqrt{ }$ \\
\hline Plum Analytics & & $\sqrt{ }$ \\
\hline Publisher Scholar Metrics & $\sqrt{ }$ & \\
\hline Qualis Livros & $\sqrt{ }$ & \\
\hline Scopus & $\sqrt{ }$ & \\
\hline Scholarly Publishers Indicators & $\sqrt{ }$ & \\
\hline
\end{tabular}

Fonte: dados da pesquisa (2019).

Os resultados obtidos a partir do mapeamento dessa última categoria analítica (Quadro 5) revelaram que, independente da tipologia de avaliação, se basear em parâmetros quantitativos, qualitativos ou mistos (Quadro 1), em $71 \%$ das iniciativas estudadas os produtos gerados (Quadro 4) possibilitam constatar o impacto científico, consubstanciando a reputação de um pesquisador, de uma publicação ou de uma editora mediante o grau de aceitação da comunidade científica. Parece oportuno salientar também que entre as iniciativas 
que possibilitam ir além do estrito impacto científico estão aquelas impulsionadas pelas métricas alternativas, extraídas de fontes não-convencionais, como as mídias sociais que, embora ampliem a possibilidade de mensurar o impacto dos livros publicados sobre a população de maneira geral, também são bastante exploradas por cientistas e pesquisadores, demonstrando o impacto social dentro da esfera científica. Essa constatação, por sua vez, parece reduzir a precisão e o alcance do impacto social tal como traduzido pelas métricas alternativas geradas nas redes sociais, pelo menos no caso dos livros acadêmicos.

Os resultados da presente pesquisa tornam evidente, por um lado, a duradoura autoridade e a forte influência dos padrões de comunicação científica próprios das ciências naturais, sustentados essencialmente pelo reconhecimento da comunidade científica, entre os modelos e sistemas de avaliação de livros acadêmicos, seja os que contabilizam a quantidade de citações recebidas, seja os que buscam a chancela de especialistas. Por outro lado, os resultados testemunham a peculiar dificuldade de expressar todo o potencial impacto que um livro publicado pode alcançar e o efetivo esforço de muitas iniciativas em capturar todos os aspectos por meio dos quais uma obra sensibiliza, surpreende e movimenta uma comunidade, uma sociedade ou uma nação, estimulando a demanda e a formulação de políticas públicas, acompanhando categorias profissionais quando se transforma em obra de referência ou influenciando o processo de ensino e aprendizagem ao ser adotado como material didático.

\section{CONSIDERAÇÕES FINAIS}

As estratégias de avaliação das atividades científicas alargaram-se consideravelmente na atualidade, examinando não somente o produto final de uma pesquisa, mas também todo o ciclo de produção da ciência: as instituições, os laboratórios, os projetos de pesquisa, os docentes e as publicações. Ao ampliar seu objeto de análise para além das publicações, a comunidade científica favoreceu a constituição de uma robusta estrutura gerencial de avaliação da ciência que, pela integração de vários atores, confere reconhecimento acadêmico, 
capital científico e credibilidade às instituições, aos pesquisadores e à sua produção.

Quanto aos mecanismos de validação dos livros acadêmicos, o panorama descortinado pelos resultados desta pesquisa apontou um progresso significativo em termos da quantidade de iniciativas que entraram em operação ao longo da última década. De acordo com as evidências obtidas, os sistemas e modelos de avaliação avançaram em consonância com a ampliação do acesso à versão digital das publicações, possibilitando a criação de caminhos alternativos aos sistemas comerciais. Nesse contexto, alguns pesquisadores e grupos de pesquisa voltaram sua atenção para a dinâmica da comunicação científica veiculada através dos livros, contribuindo para fomentar novas possibilidades de análise dessas publicações. Os projetos de avaliação de livros de origem institucional ou governamental, cujos objetivos envolvem a mensuração da produção científica de um país ou de uma área do conhecimento, também colaboraram sobremaneira para a disseminação da cultura da avaliação dos livros e para o fortalecimento dessa infraestrutura.

A constatação do predomínio de indicadores que buscam prioritariamente inferir o impacto acadêmico-científico dos livros, em detrimento de seus possíveis impactos sociais, não chegou a suscitar estranheza, já que esse padrão se repete em outras categorias de avaliação da atividade científica. Em se tratando de livros, no entanto, esse padrão mostra-se prejudicial e reducionista. Ao avaliar o impacto dos livros acadêmicos somente por uma perspectiva impede-se que seus aspectos multidimensionais, de natureza educacional, cultural e econômica, entre outros, sejam adequadamente reconhecidos e validados. Mesmo considerando essas limitações, o mapeamento das iniciativas de avaliação de livros acadêmicos que resultou desta pesquisa registra um panorama auspicioso que, a partir da pluralidade de abordagens adotadas para capturar e traduzir a qualidade das obras publicadas, favorece o reconhecimento de sua vasta contribuição à ciência e à sociedade.

\section{REFERÊNCIAS}

BRASIL. Coordenação de Aperfeiçoamento de Pessoal de Nível Superior. Diretoria de 
Avaliação. Proposta de Classificação de Livros: GT 'Qualis Livro’. Brasília, 2019.

Disponível em: http://uploads.capes.gov.br/files/classificacao_livros_ap.pdf. Acesso em: 06 fev. 2019.

CAFÉ, A. L. P. A produção científica do campo da Sociologia brasileira face aos critérios de avaliação do CNPq e da CAPES: 2007-2009. 2012. Dissertação (Mestrado em Ciência da Informação) - Instituto de Ciência da Informação, Universidade Federal da Bahia, Salvador, 2012.

CAFÉ, A. L. P.; RIBEIRO, N. M.; PONCZEK, R. L. A docilização do corpo docente na pós-graduação brasileira: um estudo sobre o modelo Capes de avaliação da produção intelectual. Curitiba: Appris, 2018.

FRANÇA, C. M. Avaliação de livros acadêmicos: mapeamento dos modelos e indicadores contemporâneos. Dissertação (Mestrado Profissional em Biblioteconomia) - Universidade Federal do Estado do Rio de Janeiro, Rio de Janeiro, 2019.

GARFIELD, E. Citation indexes for retrieval and research evaluation. In: CONSENSUS CONFERENCE ON THE THEORY AND PRACTICE OF RESEARCH EVALUATION, 7., 1996, Capri. Proceedings [...]. [S. l.: s. n.], 1996. Disponível em:

http://www.garfield.library.upenn.edu/papers/ciretreseval-capri2.pdf. Acesso em: 06 fev. 2019.

GIMÉNEZ-TOLEDO, E.; MAÑANA-RODRIGUEZ, J.; SIVERTSEN, G. Scholarly book publishing: its information sources for evaluation in the social science and humanities.

Research Evaluation, v. 26, n. 2, p. 91-101, 2017. Disponível em:

https://academic.oup.com/rev/article-abstract/26/2/91/3745099. Acesso em: 10 abr. 2018.

GIMÉNEZ-TOLEDO, E.; MAÑANA-RODRIGUEZ, J.; TEJADA-ARTIGAS, C. M.

Revisión de iniciativas nacionales e internacionales sobre evaluación de libros y editoriales.

EI Profesional de la Información, v. 24, n. 6, p. 705-716, 2015. Disponível em:

http://www.elprofesionaldelainformacion.com/contenidos/2015/nov/02_esp.pdf. Acesso em: 08 abr. 2018.

GIMÉNEZ-TOLEDO, E.; TEJADA-ARTIGAS, C.-M.; BORGES-DE-OLIVEIRA, A. El 
libro y las editoriales académicas según los investigadores brasileños de Ciencias Sociales y Humanidades. El Profesional de la Información, v. 28, n. 6, p. 1-14, 2019. Disponível em: https://revista.profesionaldelainformacion.com/index.php/EPI/article/view/epi.2019.nov.03. Acesso em: 20 nov. 2020.

GIMÉNEZ-TOLEDO, E. et al. Taking scholarly books into account: current developments in five countries. Scientometrics, v. 107, n. 2, p. 685-699, 2016. Disponível em:

https://www.researchgate.net/profile/Tim_Engels/publication/294576945_Taking_scholarly_b ooks_into_account_current_developments_in_five_European_countries/links/5700156c08aea 6b77469b593/Taking-scholarly-books-into-account-current-developments-in-five-Europeancountries.pdf. Acesso em: 11 abr. 2018.

HICKS, D. The four literatures of social science. In: MOED, H. (org.). Handbook of quantitative science and technology research. Dordrecht: Springer, 2004. p. 473-496. Disponível em:

http://yunus.hacettepe.edu.tr/ tonta/courses/spring2011/bby704/Handbook\%20of\%20Quantit ative $\% 20$ Science $\% 20$ and\%20Technology\%20Research.pdf\#page=467. Acesso em: 25 abr. 2018.

KUHN, T. S. A estrutura das revoluções científicas. 9. ed. São Paulo: Perspectiva, 2007.

LATOUR, B. A vida de laboratório: a produção dos fatos científicos. São Paulo: Relume Dumara, 1997.

LUZ, M. T. O futuro do livro na avaliação dos programas de pós-graduação: uma cultura do livro seria necessária. Interface, v. 9, n. 18, p. 631-636, 2005. Disponível em: https://www.scielosp.org/article/ssm/content/raw/?resource_ssm_path=/media/assets/icse/v9n 18/a17v9n18.pdf. Acesso em: 30 maio 2018.

MEADOWS, A. J. A comunicação científica. Brasília, DF: Briquet de Lemos, 2009.

MOMESSO, A. C.; NORONHA, D. P. Bibliométrie ou Bibliometrics: o que há por trás de um termo? Perspectivas em Ciência da Informação, v. 22, n. 2, 2017. Disponível em: https://www.scielo.br/scielo.php?script=sci_arttext\&pid=S1413-99362017000200118. Acesso em: 20 nov. 2020. 
MUELLER, S. P. M. A publicação da ciência: áreas científicas e seus canais preferenciais. Datagramazero, Rio de Janeiro, v. 6, n. 1, p. 1-13, 2005. Disponível em:

http://repositorio.unb.br/bitstream/10482/980/2/ARTIGO_PublicacaoCiencia.pdf. Acesso em: 23 maio 2018.

RODRIGUES, K. O.; ROSA, F. G.; OLIVEIRA, M.; BARROS, S. O livro no sistema de avaliação da CAPES. SCIELO 20 ANOS: Repositório, p. 1-23, 2018. Disponível em: https://repository.scielo20.org/index.php/documents/article/view/129. Acesso em: 20 nov. 2020.

SANTOS, R. N. M.; CABALLERO-RIVERO, A.; SÁNCHEZ-TARRAGÓ, N. Práticas de publicação e avaliação em Ciências Sociais e Humanidades: contradições e desafios. P2P \& Inovação, v. 4, n. 1, p. 18-34, 2018. Disponível em: http://revista.ibict.br/p2p/article/download/3982/3313/. Acesso em: 20 nov. 2020.

THOMPSON, J. W. The death of the scholarly monograph in the humanities? Citation patterns in literary scholarship. Libri, v. 52, n. 3, p. 121-136, 2002. Disponível em: https://www.researchgate.net/profile/Jennifer_Thompson15/publication/252704534_The_Dea th_of_the_Scholarly_Monograph_in_the_Humanities_Citation_Patterns_in_Literary_Scholar ship/links/00b7d53985a111c570000000.pdf. Acesso em: 26 abr. 2018.

TORRES-SALINAS, D.; ROBINSON-GARCIA, N.; GORRAIZ, J. Filling the citation gap: measuring the multidimensional impacto of the academic book at institutional level with PlumX. Scientometrics, v. 113, n. 3, p. 1371-1384, 2017. Disponível em: https://arxiv.org/ftp/arxiv/papers/1710/1710.00368.pdf. Acesso em: 11 abr. 2018.

TRZESNIAK, P. A questão do livre acesso aos artigos publicados em periódicos científicos. Em Aberto, v. 25, n. 87, p. 77-112, 2012. Disponível em: http://www.emaberto.inep.gov.br/ojs3/index.php/emaberto/article/view/2701. Acesso: 20 nov. 2020.

WHITLEY, R. Changing governance and authority relations in the public sciences. Minerva, v. 49, n. 4, p. 359-385, 2011. Disponível em: https://link.springer.com/article/10.1007/s11024011-9182-2. Acesso em: 20 nov. 2020.

WILSDON, J. et al. The metric tide: independent review of the role of metrics in research assessment and management. Bristol: Higher Education Funding Council for England, 2015. 
Disponível em: http://eprints.whiterose.ac.uk/117033/1/2015_metric_tide.pdf. Acesso em: 13 maio 2019.

ZUCCALA, A.; ROBINSON-GARCIA, N. Reviewing, indicating, and counting books for modern research evaluation systems. In: GLÄNZEL, W. et al. (org.). Springer handbook of science and technology indicators. Dordrecht: Springer Nature, 2019. p. 423-430.

Disponível em: https://arxiv.org/ftp/arxiv/papers/1807/1807.05789.pdf. Acesso em: 13 maio 2019. 